\title{
Discussing the diagnosis and prognosis with cancer patients
}

\author{
Michael Bennett, Dawn Alison
}

\begin{abstract}
Summary
Effective doctor-patient communication is an integral part of good clinical care. Telling a patient that he/she has cancer can be a daunting task. If done with empathy and sensitivity it can create an important bond between the doctor and patient. If done brusquely and without tact it can create barriers and lasting hostility. Several key steps help make the breaking of bad news easier for doctors and patients. There is not one 'right formula' but appreciation of and responsiveness to the patient's verbal and non-verbal signals are core skills which can be developed.
\end{abstract}

Keywords: communication skills, cancer

St James's University Hospital, Clinical Sciences Building, Level 08, Room 8.43a, Beckett Street, Leeds LS9 7TF, UK

M Bennett

D Alison

Accepted 14 August 1995
Cancer is a common illness with an overall lifetime incidence of one in three. It causes one in four deaths annually in the UK. Doctors are aware of the many different forms that cancer takes and that it is not inevitably fatal. The lay public, although increasingly well informed by the press and media, still tends to view a diagnosis of cancer as an imminent death sentence. Disclosing to patients that they have cancer is accepted practice now in western cultures and there is evidence in support of frank discussion about diagnosis and treatment details with cancer patients. ${ }^{1,2}$

Breaking bad news is difficult and the way in which someone receives news can leave a lasting impression on them. Patients appreciate it being done in a sensitive way and they, along with their relatives, can harbour long-term resentment if it is done badly. It is essential for doctors to prepare for the reality of breaking bad news and to look at ways of improving their skills (see boxes 1 and 2). The fact that communication ability is a skill that can be taught and improved has been demonstrated previously. ${ }^{3}$ An increasing number of medical schools now include communication skills teaching in their curricula and clinicians working closely with cancer patients can benefit from tuition in this area. ${ }^{4}$ Interactive teaching methods using videos, role play and discussion work well and there are a range of training resources available..$^{5-7}$

Receiving a diagnosis of cancer will almost inevitably be perceived as bad news. It is important to accept that bad news cannot be turned into good news. Calman suggested that quality of life could be conceptualised as the gap between a person's hopes and expectations and that of the realities of their life. ${ }^{8}$ An analogy exists when breaking bad news; the degree of bad news is proportional to the distance between the patient's perception of the situation and reality. ${ }^{9}$ The task for the doctor is to ease the patient from their perception of the situation to the reality of that situation. The skill in achieving this lies in controlling the speed of the transition in order that the patient can digest the information at his or her pace (see box 3). The larger the 'gap', the more difficult this can be. It has been argued that too quick a transition can prevent adaptation and induce denial. ${ }^{10}$

The following outline suggests an approach to discussing a diagnosis of cancer with a patient based on our own experience and recommendations of others. These steps can be adapted to a variety of clinical situations where bad news is being broken and are not designed solely for discussing the diagnosis of cancer.

\section{Preparation for the interview}

Adequate preparation and time are essential for breaking bad news in order to avoid psychological scarring for the patient. If a surgeon attempted a difficult elective operation with inadequate operating time, information, or facilities, a stormy postoperative course with the potential for long-term complications could be expected. There are direct parallels when breaking bad news.

Preparation of the patient can be started at early consultations by anticipating situations that are likely to lead to bad news being given. For example, when investigations are being organised, it is best to be realistic and honest. If a doctor suspects that the tests will confirm a clinical suspicion of malignancy (or even if malignancy is a possible differential diagnosis) it is useful to indicate this to the patient. A phrase such as "I am worried about serious disease and this test will help to exclude it" conveys this. This can also be a useful time to find out how much of the test results the patient wants to be told and whether they want a relative or friend with them at the next consultation. There is a difficult balance between alarming patients unnecessarily and falsely re-assuring them, but there is no room for platitudes or false reassurances as this could lead to a loss of confidence in the doctor at a later stage. 


\begin{tabular}{|l|}
\hline Breaking bad news \\
\hline - forms part of clinical practice \\
it is a skill that can be taught and \\
improved \\
- patients and relatives appreciate \\
discussion in a sensitive manner \\
the degree to which the news is bad \\
depends on the gap between the \\
patient's perception of the situation \\
and reality \\
\hline
\end{tabular}

Box 1

\begin{tabular}{|l|}
\hline $\begin{array}{l}\text { Benefits of learning to break } \\
\text { bad news in a skilful way }\end{array}$ \\
\hline - enables better psychological \\
adjustment by the patient \\
- reduces stress in doctors \\
- facilitates open discussion between \\
patients, their relatives and their \\
doctors \\
- empowers patients by allowing \\
them a greater say in treatment \\
decisions \\
\hline
\end{tabular}

Box 2

\begin{tabular}{l} 
Objectives of breaking bad \\
news \\
\hline remember that bad news can never \\
be good news \\
the task is to close the gap between \\
the patient's perception of the \\
situation and the reality of the \\
situation \\
the skill lies in controlling the speed \\
of the transition to fit the patient's \\
pace
\end{tabular}

Box 3

\begin{tabular}{|l|}
\hline $\begin{array}{l}\text { Preparation for the } \\
\text { interview }\end{array}$ \\
\hline - make time to do it well \\
ensure adequate and correct \\
information \\
- ensure privacy \\
introduce yourself and ascertain \\
how others in the room are related \\
to the patient \\
- sit down and make eye contact \\
if the patient is alone, allow time for \\
the relatives to be there if the \\
patient wants this, where possible \\
\hline
\end{tabular}

\section{Beginning the interview}

The doctor should avoid being rushed, though this is sometimes difficult to achieve. Reading the medical notes carefully and ensuring that the correct information is present is vital before meeting the patient. If the bad news hinges on the results of recent investigations the doctor should read the written reports personally and have them to hand. Using another person's interpretation of the reports can lead to confusion. Privacy is desirable and a separate room is best. The presence of a nurse who is involved with the patient can provide on-going support for the patient and their relatives after the bad news has been given. If the patient wishes relatives to be present during the discussion they should be given the opportunity to arrange this wherever possible (see box 4 ). There have been reports of the benefits of tape recording the bad news consultation and this may be an option in some settings. ${ }^{11,12}$

A brief introduction is helpful especially when the doctor has the unenviable task of breaking bad news to a patient that he/she has not met before. A hand shake followed by 'I am Dr Smith, the registrar who works with Dr Jones, the consultant you saw last week' enables the patient to place the doctor in context. Even if the patient and doctor have met before the patient may be too anxious to remember the doctor's name or face. It is important to find out the names and relationships of any other accompanying people who are in the room and to check whether the patient feels it is appropriate to speak freely in front of them. The doctor should sit down near the patient at roughly the same level and make eye contact. It is useful to indicate how much time is available in order to avoid fidgeting, clock-watching, or staring into the notes, which will reduce rapport.

\section{Clarifying the patient's story and coping strategy}

The patient's interpretation of their illness should be sought by asking about their symptoms, the results of tests so far and what treatment they have had. Finding out what he/she thought and felt at each stage by using open questions and waiting for an answer facilitates this process. A direct enquiry about the patient's understanding of the situation may be necessary, eg, "What do you understand from all the test results you have had?" The patient may have been falsely re-assured at a previous consultation and this is an opportunity for the doctor to judge the gap between the patient's perception and reality. Conversely, the patient may have been well prepared for the bad news such that their 'gap' is small and the task is easier.

After the patient's story has been clarified, the patient should be asked whether he/she wishes the doctor to explain further about the illness or the latest results. It is likely that he/she will reply 'yes' but occasionally may say 'I'd rather not know' in which case the doctor should respect that. Patients should not have information forced on them. The patient's coping strategy can be re-assessed later as it may change throughout their illness or even during the interview.

\section{Breaking the news}

If the patient has indicated that he/she wants more information then the doctor can proceed. Explaining slowly using congruent, non-verbal communication and a gentle but solemn voice is best. Giving a warning such as "I am afraid there is a serious problem" followed by a pause allows the patient to prepare for the news. The patient will often ask for clarification when he/she is ready to listen to more. The doctor can then give further details and although euphemisms are occasionally helpful it is always best to use the word 'cancer' at some stage to avoid confusion (see box 5). Some people believe that a patient's adjustment to his/her diagnosis is made worse by using the word 'cancer' but recent work has demonstrated that this is not the case. ${ }^{13}$

Once the bad news has been broken the doctor should check what the patient has absorbed and if necessary repeat any information that remains unclear. Diagrams, images or analogies can be used. Above all the doctor should try to take things at the patient's pace and accept that any patient will have a limited capacity to absorb details at such times. Acknowledging this and trying to make time available on another occasion is always desirable.

The use of simplified anatomical diagrams can be helpful to patients when explaining the result of investigations. Some patients appreciate being shown the radiological images during this process but, as with other aspects of breaking bad news, the doctor should first check the patient's coping strategy before displaying such images. A range exists between those who cope adequately by not allowing visual images of the cancer to come into their consciousness and those for whom active participation is very important. Images of the cancer make the disease 


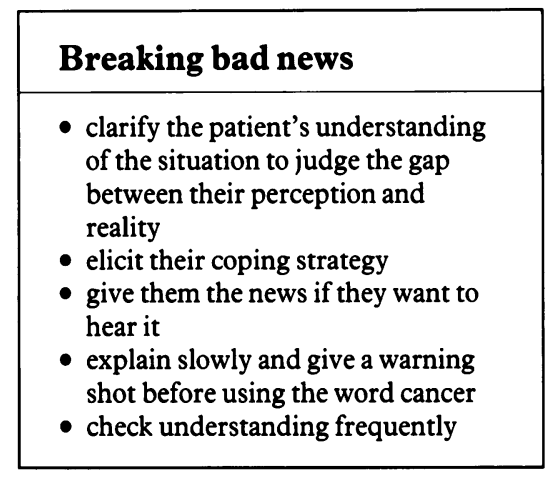

Box 5

\begin{tabular}{|l|}
\hline Responding to crying, \\
silence and anger \\
\hline - allow time for the bad news to sink \\
in and responses to occur \\
- acknowledge the patient's position \\
but avoid talking over these \\
responses to save embarrassment \\
- defensiveness or irritation are \\
unhelpful \\
the patient is usually ready to \\
re-engage with the doctor after a \\
few minutes \\
\hline
\end{tabular}

Box 6

\begin{tabular}{|l|}
\hline Discussing prognosis \\
\hline - identify any concerns that the \\
patient has for the future \\
- be realistic when discussing any \\
proposed treatments \\
- avoid giving specific time frames \\
if the patient will ultimately die of \\
their disease, offer to explain signs \\
and symptoms of deterioration but \\
avoid frightening details \\
provide examples of what the \\
patient can reasonably hope for as \\
appropriate, eg, symptom control, \\
short remission or time at home
\end{tabular}

Box 7

\begin{tabular}{|l|}
\hline Common pitfalls \\
\hline - inadequate time or information \\
- failure to elicit the patient's \\
understanding of the situation and \\
their coping strategy \\
- breaking the news at the doctor's \\
rather than the patient's pace \\
- not allowing time for responses or \\
questions \\
- platitudes and false re-assurances \\
about the future \\
- allowing collusion with relatives \\
- allowing denial to remain \\
unchallenged when it is causing \\
difficulties for the patient and their \\
relatives \\
removing all hope
\end{tabular}

more tangible; this can frighten some patients but for others it can act as a focus for their psychological battle. Conversely, some patients perceive their cancers as large and overwhelming and are greatly relieved to see that the cancer is much smaller in reality than they imagined, if this is the case.

\section{Handling the responses}

Crying Many patients cry after hearing that they have cancer. The doctor should avoid looking embarrassed and should let the patient know that this is understandable and normal behaviour. Having tissues available and avoiding speaking whilst the patient recovers his/her composure shows empathy. Some doctors feel comfortable touching the patient but not all will feel this to be appropriate. It is a matter of personal style.

Anger Anger is a fairly common response. The doctor can help discharge this by listening and letting the patient give vent to their feelings. Remaining objective and calm is vital. It helps to remember that most patients are angry at the news rather than the doctor but it is common for the messenger to be blamed. Being defensive is rarely helpful.

Silence Information may take a while to sink in. Silence can feel awkward and it is tempting to rush in with further information. The doctor should wait for a signal that the patient is ready to re-engage in their conversation. The signal might be re-making eye contact or asking further questions. The doctor can empathise, eg, "It must be very hard to take this in" but a pitfall to avoid is jumping in with false hope (see box 6).

\section{Identifying other concerns}

After breaking the news and acknowledging his/her feelings the patient's concerns should be established before any discussion of the future takes place. For example, a patient may express concern about uncontrolled symptoms such as pain. Re-assurance can be given that every effort will be made to care for them and to help control troublesome symptoms. Some patients will ask about treatment. The role of surgery, radiotherapy or chemotherapy can then be addressed but it may be preferable to do this at a later interview if the patient seems unable to take in further details. A patient may have prior knowledge of some treatments but many myths abound and so careful explanation of likely side-effects is beneficial. Quoting inflated success rates for treatments should be avoided as it can lead to anger and bitterness later if they fail.

\section{Discussing prognosis}

Questions about survival are asked by many patients. When replying to the question "How long have I got?" it is best to avoid a specific prediction. Maguire and Faulkner have highlighted the problem of patients and their relatives pacing themselves according to the time that they believe they have left. ${ }^{14}$ If the patient deteriorates sooner than anticipated they feel cheated and bitter at not achieving specific goals. An unexpected remission can be viewed as borrowed time and there may be no emotional and physical resources left to cope with this. It is better to acknowledge the uncertainty. Some patients are helped by a framework, for example, "Well it may be months rather than years, but it is unlikely to be a few weeks". This should be followed by repeating the uncertainty of the situation. Occasionally patients or relatives interpret general guidance into specific numbers of weeks or months, no matter how vague the information they have been given.

It is vital at this point to state clearly the aim of any treatment that is available and to emphasise the difference between a 'treatment' and a 'cure'. If cure is not possible then this should be conveyed sensitively.

When confronted by the question "Am I going to die?" the doctor should remember that this is a brave question and try to be honest. A reply such as "It is likely that the cancer will shorten your life"' is one option which allows the patient to ask for more details if they wish to explore this area further.

Some patients ask for guidance on how to recognise disease progression in themselves. The doctor should then describe the symptoms that they believe the patient may experience but avoid frightening details, eg, "You may notice that you are more short of breath or more easily tired". This is also another opportunity for the doctor to ask about any worries that the patient has about their future and to tackle each one separately. The memory of a relative who died in pain from cancer years ago may be a potent source of fear for the patient facing their own death (see box 7) 


\section{Case history}

A junior doctor on-call for the weekend is called to see a young woman who has had a definite carcinoma of the cervix diagnosed but has not yet been told of the diagnosis. The patient is asking what is wrong with her

The doctor could:

- read the notes carefully to ensure that the correct information is understood

- explain to the patient who they are and that they will try to help

- sit with the patient, in private if possible, and find out what she understands about her symptoms and investigations

- ask the patient if she would like the doctor to explain the results but also ask if she wants a relative to be present

- break the news if she wants to know in the manner outlined in the text

- write in the notes exactly what has been discussed

- try to speak to the consultant's team when they are back on duty. It is unlikely that the doctor will be reproached if they have responded to the patient's wishes and broken the news in a sensitive way

\section{Box 9}

\section{Case history}

A middle-aged man with lung cancer has been receiving chemotherapy but a recent $\mathrm{CT}$ scan demonstrates marked deterioration of his disease. $\mathrm{He}$ is waiting in out-patients to hear the result of the scan from the consultant.

The consultant could:

- introduce themselves if they have not met the patient before

- check what the patient understands about his condition and how he perceives the effect of the treatment so far

- warn the patient that the scan is not as good as expected

- check that he wants more explanation

- explain the scan in detail if requested

- go on to discuss alternative management and future prognosis if the patient asks, or offer to see the patient at the next out-patient clinic to let the news sink in before discussing the future

Box 10

\section{Closing the interview}

The doctor should warn the patient that they need to leave in a short while and ask the patient if they have any other worries or questions. Re-assurance about continuing support should be offered and plans made for another consultation soon. It should be remembered that coping strategies change and a patient who has been reluctant to hear bad news may be more ready to hear it at a later date. Details should be given of how the doctor can be contacted. Leaving a nurse to continue the discussion can help some patients but not all.

It is vital for teamwork that the doctor writes legibly what he/she has said to the patient in the clinical notes. For example, 'Patient told that he has stomach cancer and that it is very unlikely that he can be cured' is better and more specific than 'diagnosis and prognosis discussed'. Letters to general practitioners and referring consultants should always include a clear statement about what the patient has been told. A brief phone call to the patient's general practitioner will be much appreciated as this allows him/her to check on the patient's emotional response and provide support for the patient and family in their own home.

Most doctors find it stressful and upsetting to give bad news and whenever possible it is helpful to allow a few minutes break before going onto the next task.

\section{Difficult areas}

DENIAL

Denial can be a useful coping mechanism when a patient cannot adjust to the information given to them. It becomes a problem when it causes significant anguish for the patient or relatives and if it stops a patient from dealing with important issues for him/her and family members.

A sensitive and assertive probing of the patient's story and confronting them with the incongruence between his/her symptoms and his/her explanation may be enough to break the denial in an appropriate way. Patients can change from a position of denial to acceptance of their condition and opportunities for asking questions should be allowed at each consultation.

\section{COLLUSION WITH RELATIVES}

A conspiracy of silence can contribute to a lonely, isolated death if a patient is not given the opportunity of knowing his/her diagnosis. The strain on relatives of maintaining the 'secret' can be immense. It is important to challenge collusion to spare the patient the anxiety and distress of isolation and to avoid a complicated bereavement for relatives, who were prevented from being open with the patient by their conspiracy.

An important ethical principle is that clinical information should be discussed with a patient first, and only then with the relatives if the patient has given permission for the doctor to do so. The reality is that doctors are sometimes requested by relatives not to tell patients bad news before the doctor has even had a chance to see the patient. Usually the relative expresses fears that the patient will not be able to cope with the news and will 'give up' and they may be worried that the patient will be told bad news in a blunt manner.

In such situations, the doctor needs to be assertive whilst remaining approachable and re-assuring. Explaining the ethical position is often useful as it is not always known by the lay public. The doctor can also explain the difficulties that can be caused by a patient not knowing their diagnosis and can offer to carry out his/her discussion with the patient in as sensitive a way as possible. It is also helpful to point out to relatives that it is a natural response to become upset when hearing of a diagnosis of cancer but that many people do adjust to that knowledge. Finally, relatives should be re-assured that, if the patient indicates a clear desire not to be given bad news, this will be respected.

\section{CULTURAL DIFFERENCES}

Different cultural groups have different attitudes to disclosure of medical information. The doctor needs to be alert to this and be prepared to respect the patient's view. If there is also a language barrier and a close relative of the patient is asked to interpret, it is helpful to try and establish how comfortable the relative feels to do so. The doctor needs to be aware that the message conveyed to the patient may be altered by the interpreter because of differing traditions in what is considered acceptable practice. This is a complex area and requires more detailed analysis than is the scope of this article. 


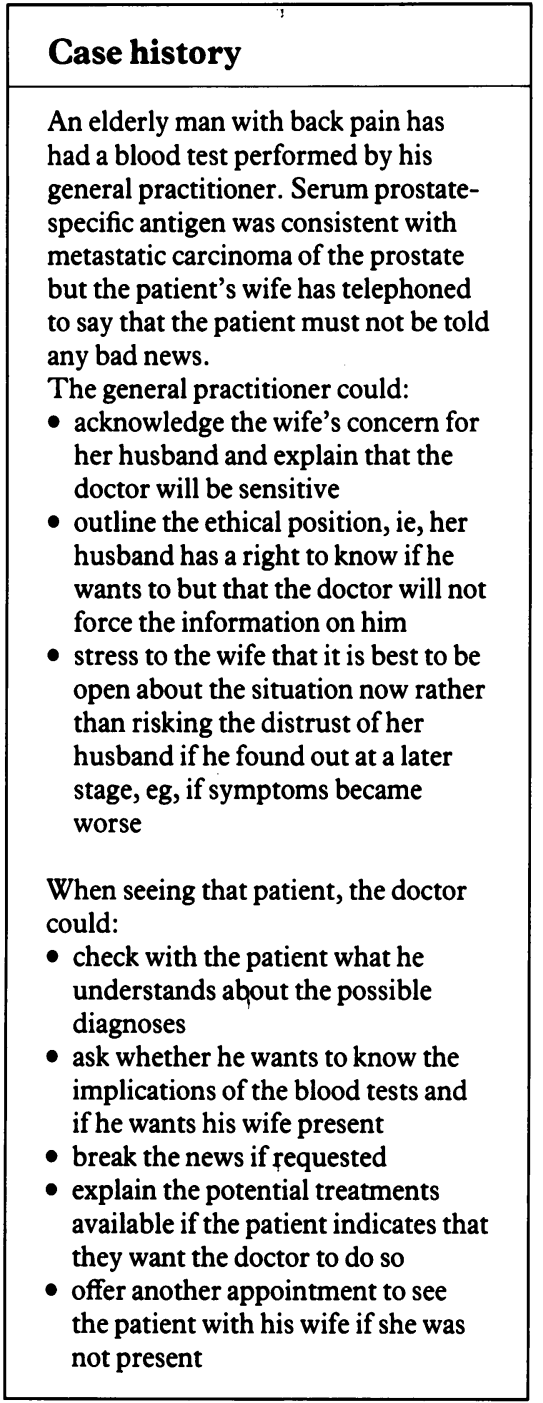

Box 11
1 Cassileth BR, Zupkis RV, Sutton-Smith $\mathrm{K}$, March V. Information and participation preferences among cancer patients. Ann Intern Med 1980; 92: 832-6.

2 Blanchard CG, Labreque MS, Ruckdeschel JC, Blanchard ED. Information and decision making preferences of hospitalized adult cancer patients. Soc Sci Med 1988; 27: 1139-45.

3 Matients. Soc Sci Med 1988; 27 . $1139-45$. Br Э Hosp Med 1990; 43: 215-6.

4 Maguire P, Faulkner A. How to do it - improve counselling skills of doctors and nurses in cancer care. $B M \mathcal{F}$ 1988; 297: 847-9.

5 McManus IC, Vincent CA, Thom S, Kidd J. How to do it - teaching communication skills to How to do it - teaching communication skills
clinical students. $B M{ }^{\prime}$ 1993; 306: 1322-7.

6 Cassidy S, Burns G, Smearden K. The cancer journey (video cassette). Professional Video Productions c/o British Gas plc, South Eastern, Katherine St, Croydon CG9 IJU.

7 Faulkner A, Maguire P. In: Talking to cancer patients and their families. Oxford: Oxford University Press, 1995; pp 187-94.

8 Calman K. Quality of life in cancer patients - an hypothesis. $\Im$ Med Ethics 1984; 10: 125-7.
9 Buckman R. How to break news. London: Pan Books, 1994; pp 11-2.

10 Maguire P, Faulkner A. How to do it - communicate with cancer patients. 1 . Handling bad news and difficult questions. $B M \mathcal{F} 1988 ; 297$ : 907-9.

11 Tattersall MH, Butow PN, Griffin AM, Dunn SM. The take home message: patients prefer consultation audiotapes to summary letters. $\mathcal{F}$ Clin Oncol 1994; 12: 1305-11.

12 Hogbin B, Fallowfield L. Getting it taped: the bad news consultation with cancer patients. $\mathrm{Br} \mathcal{F}$ Hosp Med 1989; 41: 330-3.

13 Dunn SM, Patterson PJ, Butow PN, Smart HH, McCarthy WH, Tattersall MH. Cancer by another name - a randomised trial of the effectiveness of euphemism and uncertainty in communicating with cancer patients. $f$ Clin Oncol 1993; 11: 989-96.

14 Maguire P, Faulkner A. How to do it - communicate with cancer patients. 2. Handling uncertainty, collusion and denial. $B M F 1988$; 297: 972-4 